\title{
Dihedral homology and the moon
}

\author{
Urs Frauenfelder*
}

October 15, 2018

\begin{abstract}
The equivariant homology of a twisted action of $O(2)$ on the free loop space of spheres was computed by Lodder 22] via dihedral homology. In this note we explain how this twisted action appears in the restricted three body problem after Moser regularization.
\end{abstract}

\section{Introduction}

If $N$ is a manifold, the standard action of the group $O(2)$ on the circle $S^{1}$ gives rise to an $O(2)$-action on the free loop space $\mathscr{L}_{N}=C^{\infty}\left(S^{1}, N\right)$ by

$$
g_{*} v(t)=v(g t), \quad v \in \mathscr{L}_{N}, g \in O(2), t \in S^{1} .
$$

This $O(2)$-action plays a major role in the search of closed geodesics. Indeed, if $N=S^{2}$ is the two dimensional sphere, then this $O(2)$-action is the basic reason for the existence of the three simple closed Lusternik-Schnirelman geodesics [4, 23. How dramatic the situation changes without the $O(2)$-action is impressively illustrated by the Katok examples [28]. Indeed, for nonreversible Finsler metrics the geodesic equation is only invariant under the action of $S O(2)$, and as the Katok examples illustrate, there are examples of nonreversible Finsler metrics on $S^{2}$ with only two closed geodesics.

However, if we denote by $\mathscr{L}$ the free loop space of the $n$-dimensional sphere $S^{n}=\left\{p \in \mathbb{R}^{n+1}:\|p\|=1\right\}$ we can consider the following twisted $O(2)$-action on $\mathscr{L}$. Let

$$
\rho: S^{n} \rightarrow S^{n}, \quad\left(p_{1}, p_{2} \ldots, p_{n+1}\right) \mapsto\left(-p_{1}, p_{2} \ldots, p_{n+1}\right)
$$

be the reflection at the equator. For $g \in O(2)$ set

$$
\iota(g)=\frac{1-\operatorname{det} g}{2},
$$

\footnotetext{
*Department of Mathematics and Research Institute of Mathematics, Seoul National University
} 
i.e. $\iota(g)=0$ if $g \in S O(2)$ and $\iota(g)=1$ if $g \in O(2) \backslash S O(2)$. The twisted $O(2)$-action on $\mathscr{L}$ is given for $g \in O(2)$ and $v \in \mathscr{L}$ by

$$
g_{*} v(t)=\rho^{\iota(g)} v(g t), \quad t \in S^{1} .
$$

Since $\rho$ is an involution this defines indeed an action.

For the twisted- $O(2)$ action the equivariant homology of $\mathscr{L}$ was completely computed by Lodder 22] after relating it to dihedral homology [14, 20]. Moreover, Lodder constructed in this paper a combinatorial model for the homotopy quotient which also appears in the work of Bödigheimer-Madsen [8]. In contrast to the twisted case to the authors knowledge we only partially know the equivariant homology of the standard $O(2)$-action on $\mathscr{L}$ due to work of Hingston [16.

From the point of view of closed geodesics it might seem a bit strange to look at the twisted $O(2)$-action on $\mathscr{L}$. Indeed, to take advantage of this action one has to restrict one's attention to metrics which are invariant under the reflection $\rho$. However, the purpose of this note is to explain that the twisted $O(2)$-action actually naturally arises in the study of a Hamiltonian system whose puzzling dynamics historically was one of the main motivations to investigate closed geodesics - namely the restricted problem of three bodies [5. Let us see what Poincaré himself wrote about this topic [25].

Dans mes Méthodes nouvelles de la Mécanique céleste j'ai étudié les particularités des solutions du problème des trois corps et en particulier des solutions périodiques et asymptotiques. Il suffit de se reporter à ce que j'ai écrit à ce sujet pour comprendre l'extrême complexité de ce problème; à côté de la difficulté principale, de celle qui tient au fond même des choses, il y a une foule de difficultés secondaires qui viennent compliquer encore la tâche du chercheur. Il y aurait donc intérêt à étudier d'abord un problème où on rencontrerait cette difficulté principale, mais où on serait affranchi de toutes les difficultés secondaires. Ce problème est tout trouvé, c'est celui des lignes géodésiques d'une surface.

Contrary to Poincaré's expections this note indicates that at least some aspects of the second problem are even harder than they were in the original one.

To interpret the flow of the restricted three body problem as a Hamiltonian flow on the cotangent bundle of a sphere one first has to regularize collisions. A beautiful way how two body collisions can be regularized was described by Moser [6, 24]. If one interprets $S^{n}=\mathbb{R}^{n} \cup\{\infty\}$, then the point at infinity corresponds to collisions.

Trajectories of the restricted three body problem are usually not invariant under reversal of time. Therefore the standard $O(2)$-action on $\mathscr{L}$ is of no use for the restricted three body problem. On the other hand at least since the fundamental work of Birkhoff [7] an important aspect in the study of the dynamics of the restricted three body problem is the fact that its Hamiltonian is invariant under a different antisymplectic involution. This involution extends to the regularization and hence gives rise to an $O(2)$-action on periodic trajectories of the 
restricted three body problem. In this note we explain the following observation.

Observation: After Moser regularization the $O(2)$-action on periodic trajectories of the circular restricted three body problem coincides with the twisted $O(2)$-action on $\mathscr{L}$ described above.

In [3] it was shown that in the planar case, i.e. $n=2$, below the first critical value, the bounded components of the energy hypersurfaces of the regularized restricted three body problem in $T^{*} S^{2}$ are fiberwise starshaped. This implies that their symplectic homology is well defined and coincides with the loop space homology of $S^{2}$ [1, 26, 27]. In particular, if one looks at the $O(2)$-equivariant symplectic homology defined as in 9, one gets the following Corollary if one combines Lodder's computation [22] with the Observation.

Corollary: Below the first critical value the $O(2)$-equivariant symplectic homology for each of the bounded components of the regularized circular planar restricted 3-body problem is given by the following formula

$$
S H_{*}^{O(2)}=H_{*}(B O(2)) \oplus \bigoplus_{m=1}^{\infty} H_{*}\left(D_{m} ; \mathbb{Z}[1]^{\otimes m}\right) .
$$

Remark: It is conceivable that the methods in [3] can also be used to show that in the spatial case of the restricted three body problem, i.e. $n=3$, below the first critical value the bounded components of the regularized energy hypersurface are still fiberwise starshaped in $T^{*} S^{3}$. Hence the $O(2)$ equivariant symplectic homology would still be well defined and could be computed as in the planar case to be $S H_{*}^{O(2)}=H_{*}(B O(2)) \oplus \bigoplus_{m=1}^{\infty} H_{*}\left(D_{m} ; \mathbb{Z}[2]^{\otimes m}\right)$.

Remark: It is an open question if the bounded components of the energy hypersurfaces for the regularized restricted three body problem are also fiberwise convex. If this were true, then after regularization trajectories could be interpreted as Finsler geodesics for a (nonreversible) Finsler metric. In this case symplectic homology in the Corollary above could be replaced by the Morse homology of a Finsler metric. It was shown in 12 that in the special case of the rotating Kepler problem, i.e. the case were the mass of the moon becomes zero, fiberwise convexity actually holds.

This note is organized as follows. In Section 2 we recall Lodder's computations of the equivariant homology of the loop space of a sphere for the twisted $O(2)$-action. In Section 3 we explain how the Kepler flow can be interpreted as the geodesic flow of a round sphere after Moser regularization. In Section 4 we explain the restricted three body problem and its Moser regularization. In Section 5 we explain how Birkhoff's involution extends to the Moser regularization and prove the Observation and its Corollary.

Acknowledgements: The author would like to thank Nancy Hingston for draw- 
ing his attention to Lodder's paper [22. This research was partially supported by the Basic Research fund 2010-0007669 funded by the Korean government.

\section{Dihedral homology}

To the authors knowledge historically the first complete computation of the $S O(2)$-equivariant homology of free loop spaces of spheres appeared in the paper by Carlsson and Cohen [11 and relied on deep results from cyclic homology. Before the paper by Carlsson and Cohen partial computations were achieved by Hingston [16] using Morse theory. An interesting Morse theoretic interpretation of the result by Carlsson and Cohen as well as an alternative derivation of their results can be found in 17 .

Since the scheme of Lodders computation of the twisted $O(2)$-equivariant homology of spheres follows the scheme of Carlsson and Cohen we first recall their computation. It was proved by Burghelea-Fiedorowicz, Goodwillie and Jones that if $N$ is a connected manifold and $\mathscr{L}_{N}$ the free loop space of $N$, then

$$
H_{*}^{S O(2)}\left(\mathscr{L}_{N}\right)=H C_{*}\left(C_{*}(\Omega N)\right) .
$$

Here $\Omega N$ is the Moore loop space of $N$ which is a group like topological monoid, $C_{*}(\Omega N)$ is the differential graded algebra of singular chains of $\Omega N$, and $H C_{*}$ is the cyclic hyperhomology of this differential graded algebra. Strictly speaking the paper of Jones [18] relates the equivariant loop space homology to a variant of cyclic hyperhomology applied to the differential graded algebras of cochains on $N$. How the two approaches are related is explained in [19].

If $n \geq 2$, then the Pontryagin ring of $\Omega S^{n}$ is given by

$$
H_{*}\left(\Omega S^{n}\right)=\mathbb{Z}[x], \quad|x|=n-1 .
$$

If we think of $H_{*}\left(\Omega S^{n}\right)$ as a differential graded algebra with trivial differential, then we can construct a quasiisomorphism between $H_{*}\left(\Omega S^{n}\right)$ and $C_{*}\left(\Omega S^{n}\right)$ by mapping $x$ to a representative of it. Since cyclic hyperhomology is invariant under quasiisomorphism we conclude for $\mathscr{L}=\mathscr{L}_{S^{n}}$ that

$$
H_{*}^{S O(2)}(\mathscr{L})=H C_{*}(\mathbb{Z}[x]), \quad|x|=n-1 .
$$

The latter was computed by Loday and Quillen 20, 21] to be

$$
H_{*}^{S O(2)}(\mathscr{L})=H_{*}(B S O(2)) \oplus \bigoplus_{m=1}^{\infty} H_{*}\left(\mathbb{Z}_{m} ; \mathbb{Z}[n-1]^{\otimes m}\right)
$$

where $B S O(2) \cong C P^{\infty}$ is the classifying space of the group $S O(2)$.

Thanks to the theory of crossed simplicial groups due to Fiedorowicz and Loday [14, there are analogons of (2) for more general actions then circle actions. In particular,

$$
H_{*}^{O(2)}(\mathscr{L})=H D_{*}\left(C_{*}\left(\Omega S^{n}\right)\right)
$$


where on the righthand side we have dihedral hyperhomology [13]. This is actually true for both the standard and the twisted $O(2)$-action on $\mathscr{L}$, where we have to note that the action of the dihedral group on the Hochschild complex of $C_{*}\left(\Omega S^{n}\right)$ differs in both cases. For a notation which keeps track of the actions we refer again to Dunn's paper [13].

However, just for the twisted action we can identify the righthand side of (3) with the dihedral homology of the Pontrjagin ring of $\Omega S^{n}$. First pick a basepoint on $S^{n}$ and identify $S^{n}$ with the reduced suspension $S^{n}=\Sigma S^{n-1}=S^{n-1} \wedge S^{1}$. Consider the map $\alpha: S^{n-1} \rightarrow \Omega S^{n}$ given for $p \in S^{n-1}$ by

$$
\alpha(p)(t)=(p, t) \in S^{n-1} \wedge S^{1}, \quad t \in[0,1] .
$$

Choose a cycle $\sigma \in C_{n-1} S^{n-1}$ representing a homology class $[\sigma]$ which generates $H_{n-1}\left(S^{n-1}\right) \cong \mathbb{Z}$. Then $\alpha_{*}[\sigma] \in H_{n-1}\left(\Omega S^{n}\right)$ is a generator of the Pontrjagin ring. The cycle $\alpha_{*} \sigma \in C_{n-1}\left(\Omega S^{n}\right)$ is invariant, if one simultaneously switches the suspension coordinate as well as the direction of a loop in $\Omega S^{n}$. Hence for the twisted $O(2)$-action on $\mathscr{L}$ one gets from (3)

$$
H_{*}^{O(2)}(\mathscr{L})=H D_{*}(\mathbb{Z}[x]), \quad|x|=n-1 .
$$

The dihedral homology of $\mathbb{Z}[x]$ was computed by Lodder in 22 to be

$$
H_{*}^{O(2)}(\mathscr{L})=H_{*}(B O(2)) \oplus \bigoplus_{m=1}^{\infty} H_{*}\left(D_{m} ; \mathbb{Z}[n-1]^{\otimes m}\right)
$$

where $D_{m}$ is the dihedral group of order $2 m$.

Remark: The computations of Carlsson and Cohen [11 as well as of Lodder 22 which we recalled for spheres actually apply more generally to suspensions.

\section{Moser regularization}

The Hamiltonian for the Kepler problem $H: T^{*}\left(\mathbb{R}^{n} \backslash\{0\}\right)=\left(\mathbb{R}^{n} \backslash\{0\}\right) \times \mathbb{R}^{n} \rightarrow \mathbb{R}$ is given by

$$
H(q, p)=\frac{1}{2}|p|^{2}-\frac{1}{|q|}
$$

For negative energy values trajectories of the Hamiltonian flow are either collision orbits are project to ellipses in position space. In particular, for a dense set of trajectories the flow is periodic and the only nonperiodic orbits are collision orbits. Two body collisions can always be regularized and in 24 Moser found a way to embed a reparametrization of the Kepler flow into the geodesic flow of the round metric of $S^{n}$. In particular, after regularization the flow becomes completely periodic.

Moser's method is most nicely illustrated for the energy value $c=-\frac{1}{2}$. Consider the Hamiltonian $K:\left(\mathbb{R}^{n} \backslash\{0\}\right) \times \mathbb{R}^{n} \rightarrow \mathbb{R}$ defined by

$$
K(q, p)=|q|\left(H(q, p)+\frac{1}{2}\right)+1=\frac{1}{2}\left(|p|^{2}+1\right)|q| .
$$


Note that

$$
\Sigma:=H^{-1}\left(-\frac{1}{2}\right)=K^{-1}(1) .
$$

For a point $(q, p) \in \Sigma$ the Hamiltonian vector fields satisfy

$$
X_{K}(q, p)=|q| X_{H}(q, p) .
$$

Since $|q|$ never vanishes on the energy hypersurface $\Sigma$, the flow of $X_{K}$ restricted to $\Sigma$ is just a reparametrization of the flow of $X_{H}$ on $\Sigma$. Now switch in your mind the roles of $q$ and $p$ and think of $p$ as the base coordinate and $q$ as the fiber coordinate. Moreover, think of $\mathbb{R}^{n}$ as the chart of $S^{n}$ obtained via stereographic projection. Then $K(q, p)$ is precisely the length of the cotangent vector $q \in T^{*} S^{n}$ for the round metric on $S^{n}$. Note further that the switch of base and fiber coordinates $(q, p) \mapsto(-p, q)$ is a symplectomorphism from $T^{*} \mathbb{R}^{n}$ to $T^{*} \mathbb{R}^{n}$. Hence the Hamiltonian flow of $K$ embeds into the geodesic flow of the round metric on $S^{n}$ and therefore after reparametrization the Kepler flow as well.

If one thinks of the sphere as $S^{n}=\mathbb{R}^{n} \cup\{\infty\}$, then the point at infinity corresponds to collisions. Here it is useful to remember that we switched the roles of position and momentum coordinates. Indeed, at collisions the original momentum explodes while the original position coordinate remains bounded.

If the energy value $c$ is negative but not necessarily equal to $-\frac{1}{2}$ we still get the geodesic flow on $S^{n}$ after conjugating with an additional diffeomorphism in the chart obtained via stereographic projection. Indeed, for negative $c$ set

$$
K_{c}(q, p)=|q|(H(q, p)-c)+1=\frac{1}{2}\left(|p|^{2}+|c|\right)|q| .
$$

Consider the symplectomorphism $\phi: T^{*} \mathbb{R}^{n} \rightarrow T^{*} \mathbb{R}^{n}$ given by

$$
\phi(p, q)=\left(\sqrt{|c|} p, \frac{q}{\sqrt{|c|}}\right), \quad(p, q) \in T^{*} \mathbb{R}^{n} .
$$

Then we get

$$
\phi^{*} K_{c}(p, q)=\frac{\sqrt{|c|}}{2}\left(|p|^{2}+1\right)|q|
$$

which up to a constant conformal factor is again the length of the cotangent vector $q$ for the standard round metric on $S^{n}$ in the chart obtained by stereographic projection.

\section{The restricted three body problem}

In the restricted three body problem one considers two massive bodies, the primaries, and a massless body which is attracted by the two primaries according to Newton's law of gravitation. We refer to the primaries as the earth and moon and to the massless body as the satellite. Since the satellite is assumed to be 
massless it does not affect the motion of the two primaries. Hence the motion of the two primaries is governed by Kepler's laws. In the circular restricted three body problem one assumes in addition that the two primaries move along circles around their common center of mass. One further distinguishes between the planar case, where the satellite is supposed to move in the eccliptic, i.e. the plane spanned by earth and moon, and the spatial case where the satellite is supposed to move in three dimensional space. Mathematically the problem makes sense in any dimension greater or equal to two and we therefore describe the Hamiltonian for the movement of the satellite in a space of arbitrary dimension $n \geq 2$.

After scaling the total mass to be one and excluding the case that the mass of the earth vanishes there is a $\mu \in[0,1)$ such that the mass of the moon equals $\mu$ and the mass of the earth equals $1-\mu$. Let $\left\{e_{1}, \cdots, e_{n}\right\}$ be the standard base of $\mathbb{R}^{n}$. Scaling the distance of the earth and the moon to one, translating the center of mass to the origin and applying a suitable orthogonal transformation in the inertial system the position of earth $E_{i}(t)$ and moon $M_{i}(t)$ at time $t$ are given by

$$
E_{i}(t)=\mu \cos (t) e_{1}+\mu \sin (t) e_{2}, \quad M_{i}(t)=-(1-\mu) \cos (t) e_{1}-(1-\mu) \sin (t) e_{2} .
$$

The Hamiltonian for the satellite in the inertial system

$$
H_{t}^{i}: T^{*}\left(\mathbb{R}^{n} \backslash\left\{E_{i}(t), M_{i}(t)\right\}\right) \rightarrow \mathbb{R}
$$

is given by kinetic and potential energy

$$
H_{t}^{i}(q, p)=\frac{1}{2}|p|^{2}-\frac{1-\mu}{\left|q-E_{i}(t)\right|}-\frac{\mu}{\left|q-M_{i}(t)\right|} .
$$

Note that since the earth and moon are moving in the inertial system this Hamiltonian is not autonomous, with, alas, even a time dependent domain of definition. In particular, it is not preserved along trajectories of the satellite. To improve this unpleasant situation we transform the system form inertial to rotating coordinates. In the rotating coordinate system the positions of the earth and moon are fixed

$$
E=\mu e_{1}, \quad M=-(1-\mu) e_{1} .
$$

The transition from the inertial to the rotating coordinate system involves a time dependent transformation, namely we have to rotate the $\left(e_{1}, e_{2}\right)$-plane in $\mathbb{R}^{n}$. The infinitesimal generator of this rotation is given by angular momentum

$$
L(q, p)=p_{1} q_{2}-p_{2} q_{1}
$$

Hence the Hamiltonian $H: T^{*}\left(\mathbb{R}^{n} \backslash\{E, M\}\right) \rightarrow \mathbb{R}$ in the rotating coordinate system becomes [2]

$$
H(q, p)=\frac{1}{2}|p|^{2}-\frac{1-\mu}{|q-E|}-\frac{\mu}{|q-M|}+L(q, p) .
$$


Note that in the rotating coordinate system the Hamiltonian is autonomous and therefore preserved under its Hamiltonian flow. This would in general not be true for the elliptic restricted three body problem where the primaries are allowed to move on ellipses. For that reason it is important to restrict to the circular case. Since this observation goes back to Jacobi the integral of motion $-2 H$ is usually referred to as the Jacobi integral.

Introducing the effective potential $U: \mathbb{R}^{n} \backslash\{E, M\} \rightarrow \mathbb{R}$ given by

$$
U(q)=-\frac{1-\mu}{|q-E|}-\frac{\mu}{|q-M|}-\frac{1}{2}\left(q_{1}^{2}+q_{2}^{2}\right)
$$

the Hamiltonian $H$ can be rewritten as

$$
H(q, p)=\frac{1}{2}\left(\left(p_{1}+q_{2}\right)^{2}+\left(p_{2}-q_{1}\right)^{2}+\sum_{i=3}^{n} p_{i}^{2}\right)+U(q) .
$$

Note that the footpoint projection $\pi: T^{*}\left(\mathbb{R}^{n} \backslash\{E, M\}\right) \rightarrow \mathbb{R}^{n} \backslash\{E, M\}$ restricts to a bijection between critical points

$$
\Pi=\left.\pi\right|_{\operatorname{crit}(H)}: \operatorname{crit}(H) \rightarrow \operatorname{crit}(U) .
$$

If $\mu \neq 0$, i.e. the mass of the moon does no vanish, there are five critical points of $U$ for every massratio, usually referred to as Lagrange points. The first three Lagrange points $L_{1}, L_{2}$ and $L_{3}$ are saddle points of $U$ and are collinear with the earth and moon, $L_{1}$ lies between $E$ and $M, L_{2}$ lies to the right of $E$, and $L_{3}$ lies to the left of $M$. The Lagrange points $L_{4}$ and $L_{5}$ are maxima of $U$. They lie in the $\left(e_{1}, e_{2}\right)$-plane and together with each of the primaries span an equilateral triangle. In the limiting case where $\mu$ is zero, i.e. the rotating Kepler problem, the critical set of $U$ consists of the circle of radius one around the origin in the $\left(e_{1}, e_{2}\right)$-plane.

For an energy value $c \in \mathbb{R}$ abbreviate by $\Sigma_{c}=H^{-1}(c)$ the energy hypersurface of $H$. The Hill's region is defined to be

$$
\mathcal{K}_{c}=\pi\left(\Sigma_{c}\right)=\left\{q \in \mathbb{R}^{n} \backslash\{E, M\}: U(q) \leq c\right\} .
$$

When $\mu \in(0,1)$ it can be shown that $L_{1}$ has the smallest action of all five critical points of $U$. If $c<U\left(L_{1}\right)=H\left(\Pi^{-1}\left(L_{1}\right)\right)$ the Hill's region has three connected components. Two of these connected components are bounded. One bounded component contains the earth in its closure and one bounded component contains the moon in its closure. The third region is unbounded. We denote by $\mathcal{K}_{c}^{E}$ the bounded component which contains $E$ in its closure and abbreviate

$$
\Sigma_{c}^{E}=\left\{(q, p) \in \Sigma_{c}: q \in \mathcal{K}_{c}^{E}\right\} .
$$

If $\mu=0$ there is a single critical value of $U$ which can be computed to be $-\frac{3}{2}$. For $c<-\frac{3}{2}$ the Hills region has one bounded and one unbounded component. The bounded component contains $E$ in its closure and we define $\mathcal{K}_{c}^{E}$ and $\Sigma_{c}^{E}$ as before. For later reference let us abbreviate

$$
\kappa=\left\{\begin{array}{cc}
U\left(L_{1}\right) & \text { if } \mu \in(0,1) \\
-\frac{3}{2} & \text { if } \mu=0
\end{array}\right.
$$


to be the first critical value of $H$.

We denote by $\varphi: T^{*} \mathbb{R}^{n} \rightarrow T^{*} \mathbb{R}^{n}$ the symplectomorphism $(q, p) \mapsto(-p, q-E)$ and by $\iota: T^{*} \mathbb{R}^{n} \rightarrow T^{*} S^{n}$ the inclusion obtained by interpreting $\mathbb{R}^{n}$ as the chart of $S^{n}$ under stereographic projection. We define the Moser regularization of $\Sigma_{c}^{E}$ to be

$$
\bar{\Sigma}_{c}^{E}=\operatorname{cl}\left(\iota \varphi\left(\Sigma_{c}^{E}\right)\right) \subset T^{*} S^{n}
$$

where cl means closure. Recall that a hypersurface $\Sigma \subset T^{*} S^{n}$ is called fiberwise starshaped, if for each $p \in S^{n}$ the intersection $\Sigma \cap T_{p}^{*} S^{n}$ bounds a starshaped domain in $T_{p}^{*} S^{n}$. The following result was proved in [3].

Theorem 4.1 (Albers-Frauenfelder-van Koert-Paternain) In the planar case, i.e. $n=2$, if $c<\kappa$ then $\bar{\Sigma}_{c}^{E}$ is a fiberwise starshaped hypersurface in $T^{*} S^{2}$.

Remark: Of course this holds also for the regularization around the moon. Just replace $\mu$ by $1-\mu$ and the moon becomes the earth while the earth becomes the moon.

Remark: We expect that the same result also holds in higher dimensions, although we have not checked the details.

\section{The Birkhoff involution}

We write $q=\left(q_{1}, q_{2}, q^{3}\right)$ with $q^{3} \in \mathbb{R}^{n-2}$ for a vector in $\mathbb{R}^{n}$ The Hamiltonian $H$ of the circular restricted three body problem is invariant under the antisymplectic involution $B: T^{*} \mathbb{R}^{n} \rightarrow T^{*} \mathbb{R}^{n}$ given by

$$
B\left(q_{1}, q_{2}, q^{3}, p_{1}, p_{2}, p^{3}\right)=\left(q_{1},-q_{2},-q^{3},-p_{1}, p_{2}, p^{3}\right) .
$$

Indeed, since $H$ is invariant under $B$ but the symplectic form $\omega=\sum_{i=1}^{n} d q_{i} \wedge d p_{i}$ on $T^{*} N$ is antiinvariant, the Hamiltonian vector field $X_{H}$ of $H$ is antiinvariant as well. In particular, if $w \in C^{\infty}\left(\mathbb{R}, T^{*} \mathbb{R}^{n}\right)$ is a trajectory of the Hamiltonian flow of $H$, i.e. a solution of the ODE $\partial_{t} w=X_{H}(w)$ and $R w \in C^{\infty}\left(\mathbb{R}, T^{*} \mathbb{R}^{n}\right)$ is defined to by

$$
R w(t)=B(w(-t))
$$

then $R w$ is still a trajectory of the Hamiltonian flow. Periodic orbits which are invariant under the involution $R$ are called symmetric periodic orbits and they played at least since the work of Birkhoff [7 a major role in the study of the dynamics of the restricted three body problem.

We are now in position to proof the Observation we mentioned in the Introduction.

Proof of the Observation: First we note that we can extend $B$ to the Moser regularization as follows. Let $\rho: S^{n} \rightarrow S^{n}$ be the reflection along the equator as in (1). The adjoint of its differential $d^{*} \rho: T^{*} S^{n} \rightarrow T^{*} S^{n}$ is a symplectic involution which commutes with the antisymplectic involution $I: T^{*} S^{n} \rightarrow T^{*} S^{n}$ 
whose restriction to each fibre is given by $\left.I\right|_{T_{p}^{*} S^{n}}=-\left.\mathrm{id}\right|_{T_{p}^{*} S^{n}}$. Their composition $I \circ d^{*} \rho$ is an antisymplectic involution on $T^{*} S^{n}$ which extends $B$. Thinking of $O(2)$ as the semidirect product $O(2)=S O(2) \rtimes \mathbb{Z}_{2}$ we get an $O(2)$-action on the free loop space of $T^{*} S^{n}$ as follows. For the subgroup $S O(2)$ in $O(2)$ the action is just given by rotation of the domain. If $r$ is the generator of $\mathbb{Z}_{2}$ and $w \in \mathscr{L}_{T^{*} S^{n}}$, then the action (id, $\left.r\right) \in S O(2) \rtimes \mathbb{Z}_{2}$ is given by (id, $\left.r\right)_{*} w=R w$. If we look at the shadow of $w$ in the free loop space of $S^{n}$ after applying the footpoint projection $\pi: T^{*} S^{n} \rightarrow S^{n}$ we precisely recover the twisted $O(2)$-action on $\mathscr{L}$ from the introduction. This finishes the proof of the Observation.

We finally prove the Corollary from the introduction.

Proof of the Corollary: By Theorem $4.1 \bar{\Sigma}_{c}^{E}$ bounds a Liouville domain $D_{c}$ in $T^{*} S^{2}$ which is isotopic to the unit disk bundle in $T^{*} S^{2}$. Hence by a theorem of Abbondandolo-Schwarz, Salamon-Weber, and Viterbo [1, 26, 27, its symplectic homology computes the loop space homology of $S^{2}$. Looking at the $O(2)$-invariant symplectic homology [9] we get

$$
S H_{*}^{O(2)}\left(D_{c}\right)=H_{*}^{O(2)}\left(\mathscr{L}_{S^{2}}\right)
$$

where by the Observation the $O(2)$-action is the twisted $O(2)$-action on the loop space of $S^{2}$. Lodder's computations (4) now imply the Corollary.

\section{References}

[1] A. Abbondandolo, M. Schwarz, On the Floer homology of cotangent bundles, Comm. Pure Appl. Math. 59 (2006), 254-316.

[2] R.Abraham, J.Marsden, Foundations of Mechanics, 2nd ed. AddisonWesley, New York (1978).

[3] P. Albers, U. Frauenfelder, O. van Koert, G. Paternain, The contact geometry of the restricted 3-body problem, Comm. Pure Appl. Math. 65, (2012), no. 2, 229-263.

[4] W. Ballmann, Der Satz von Lusternik-Schnirelmann, Bonner Math. Schriften 102 (1978).

[5] J. Barrow-Green, Poincaré and the three body problem, History of Mathematics, 11 (1997).

[6] E. Belbruno, Capture Dynamics and Chaotic Motions in Celestial Mechanics, Princeton University Press (2004).

[7] G. Birkhoff, The restricted problem of three bodies, Rend. Circ. Matem. Palermo 39 (1915), 265-334.

[8] C. Bödigheimer, I. Madsen, Homotopy quotients of mapping spaces and their stable splitting, Quart. J. Math. Oxford (2), 39 (1988), 401-409. 
[9] F. Bourgeois, A. Oancea, The Gysin exact sequence for $S^{1}$-equivariant symplectic homology, arXiv:0909.4526.

[10] D. Burghelea, Z. Fiedorowicz, Cyclic homology and algebraic K-theory of spaces II, Topology 25 (1986), 303-317.

[11] G. Carlsson, R. Cohen, The cyclic groups and the free loop space, Comment. Math. Helv. 62 (1987), 423-449.

[12] K. Cieliebak, U. Frauenfelder, O.van Koert, The Cartan geometry of the rotating Kepler problem, arXiv:1110.1021

[13] G.Dunn, Dihedral and quaternionic homology and mapping spaces, Ktheory 3 (1989), 141-161.

[14] Z. Fiedorowicz, J. Loday, Crossed simplicial groups and their associated homology, Trans. Amer. Math. Soc., 326 (1991), 57-87.

[15] T. Goodwillie, Cyclic homology, derivations and the free loop space, Topology 24 (1985), 187-215.

[16] N. Hingston, Equivariant Morse theory and closed geodesics, J. Diff. Geom., 19 (1984), 85-116.

[17] N.Hingston, An Equivariant Model for the Free Loop space of $S^{N}$, Amer. Jour. of Math., 114 (1992), no. 1, 139-155.

[18] J. Jones, Cyclic homology and equivariant homology, Invent. Math. 87 (1987), 403-423.

[19] J. Jones, J. McCleary, Hochschild homology, cyclic homology, and the cobar construction, Adams Memorial Symposium on Algebraic Topology, London Math. Soc. Lecture Note Ser., 175 (1992), 53-65.

[20] J. Loday, Cyclic homology, Berlin, Heidelberg, New York: Springer-Verlag (1992).

[21] J. Loday, D. Quillen, Cyclic homology and the Lie algebra homology of matrices, Comment. Math. Helvetici 59 (1984), 565-591.

[22] G. Lodder, Dihedral homology and the free loop space, Proc. London Math. Soc. (3) 60 (1990), no. 1, 201-224.

[23] L.Lusternik, L.Schnirelmann, Sur le problème des trois géodésiques fermées sur les surfaces de genre 0, C. R. Acad. Sci. Paris 189 (1929), 269271.

[24] J. Moser, Regularization of Kepler's problem and the averaging method on a manifold, Comm. Pure Appl. Math., 23 (1970), 609-636.

[25] H.Poincaré, Sur les lignes géodésiques des surfaces convexes, Trans. Amer. Math. Soc., 17 (1909), 237-274. 
[26] D. Salamon, J. Weber, Floer homology and the heat flow, Geom. Funct. Anal. 16 (2006), 1050-1138.

[27] C. Viterbo, Functors and computations in Floer homology with applications. II, Preprint Université Paris-Sud no 98-15 (1998).

[28] W. Ziller, Geometry of the Katok examples, Ergod. Th. \& Dynam. Syst., 3 (1982), 135-157. 